\title{
Phase-covariant quantum benchmarks
}

\author{
J. Calsamiglia, M. Aspachs, R. Muñoz-Tapia, and E. Bagan \\ Grup de Física Teòrica, Universitat Autònoma de Barcelona, Bellaterra, 08193 Barcelona, Spain
}

(Received 31 July 2008; published 8 May 2009)

\begin{abstract}
We give a quantum benchmark for teleportation and quantum storage experiments suited for pure and mixed test states. The benchmark is based on the average fidelity over a family of phase-covariant states and certifies that an experiment cannot be emulated by a classical setup, i.e., by a measure-and-prepare scheme. We give an analytical solution for qubits, which shows important differences with standard state estimation approach, and compute the value of the benchmark for coherent and squeezed states, both pure and mixed.
\end{abstract}

DOI: 10.1103/PhysRevA.79.050301

PACS number(s): 03.67.Hk, 03.67.Mn

\section{INTRODUCTION}

A central question in quantum information theory is whether a particular quantum protocol can be realized with the same efficiency by classical means; ultimately, quantum information stands on the advantage of quantum over classical systems in performing certain tasks. This question is relevant in experimental implementations of such protocols as they become imperfect in real unavoidably noisy experiments. It is then essential to assess whether the same experimental result could have been obtained by using only classical (less costly) resources. So, for example, in a particular teleportation experiment-which involves generating entanglement, performing complicated Bell measurements, fighting decoherence, etc. - one may ask whether the same goal, i.e., mapping the state of a system onto a second system at a different space-time location, could have been achieved by measuring the quantum state of the first system, transmitting the collected information, and preparing the state of the second accordingly. In this Rapid Communication, we propose and calculate quantum benchmarks that certify that a certain implementation of teleportation cannot be realized classically or more precisely by a measure-and-prepare strategy. Of course, these benchmarks also apply to any protocol that can be understood as a realization of the identity channel, e.g., quantum storage. Our benchmarks are based on phase-covariant families of states, and thus they are computationally manageable and experimentally feasible (shifting phases is straightforward in, e.g., optical experiments). Last but not least, our benchmarks apply when test states are mixed, which is the standard situation in experiments. Previously proposed quantum benchmarks [1-3] were either restricted to pure states or were not strict bounds since they were based on suboptimal classical strategies (see footnote 15 in [3]). We give rigorous quantum benchmarks for both pure and mixed Gaussian states that can be immediately applied to current experiments on continuous-variable (CV) systems, such as optical fields and atomic ensembles [4,5]. We note here that there are types of criteria to establish the success of CV teleportation that are not based on a single parameter, most notably [6,7], here however we focus on fidelity based benchmarks [8].

\section{BACKGROUND AND METHODS}

The literature on quantum benchmarks originated in the context of $\mathrm{CV}$ teleportation experiments, the first of which was performed in 1998 by Furusawa et al. [4]. In this experiment, optical coherent states were teleported using squeezedstate entanglement. As benchmark, they used the average fidelity, $\mathcal{F}$, that can be attained without entanglement (with the EPR beams replaced by the vacuum). Braunstein et al. [8] proposed a more rigorous benchmark for CV teleportation. They considered the fidelity between an input state $\left|\psi_{\text {in }}\right\rangle$ and the corresponding state, $\rho_{\text {out }}$, outputted from a measureand-prepare channel, i.e., $\rho_{\text {out }}=\Sigma_{\chi} p\left(\chi \mid \psi_{\text {in }}\right) \rho_{\chi}$, where $\rho_{\chi}$ is the reconstructed or guessed state based on outcome $\chi$ of the measurement and $p\left(\chi \mid \psi_{\text {in }}\right)$ is the conditional probability of obtaining $\chi$ given that the signal state is $\left|\psi_{\text {in }}\right\rangle$. Their benchmark is given by the maximum fidelity averaged over a conveniently chosen set $\Omega$ of input states. It should be stressed that the choice of $\Omega$ plays a significant role. It should contain necessarily more than one state (otherwise a trivial classical strategy achieves fidelity 1 ). The maximum fidelity tends to decrease with the size of the test family. When the input states are drawn from a whole $d$-dimensional Hilbert space, the optimal fidelity is known to be $\mathcal{F}=2 /(d+1)$ [9]. In the case of CV systems, $d \rightarrow \infty$, this would mean that any nonzero fidelity would certify that quantum resources are being used. However, it is utterly unrealistic to assume that one can test the channel with such a large family of input states. In order to have realistic and practical thresholds, Braunstein et $a l$. [8] chose $\Omega$ to be the set of coherent states with normally distributed amplitude around $|\alpha|=0$ and a fixed given variance and gave a classical strategy that was recently proved to be optimal by Hammerer et al. [1].

Adesso and Chiribella [3] very recently proposed a quantum benchmark suited for pure single-mode squeezed states and derived upper and lower bounds for squeezed thermal states. Their quantum benchmark is taken to be the maximum averaged fidelity over all the outcomes $\chi$ of an optimal measurement and over an ensemble $\Omega$ of input states $\rho_{\text {in }}(r)$ whose squeezing parameter $r$ is distributed with a given prior probability (in [2] a benchmark for pure squeezed states drawn from a microcanonical ensemble was considered). In this approach, (i) the output of the measure-and-prepare channel is compared to the input for each particular measurement outcome $\chi$ and then averaged and (ii) the output state is assumed to belong to the input family, i.e., is of the form $\rho\left(r_{\chi}\right)$. As we will see this choice of output state is typically suboptimal and, hence, the corresponding fidelity might not be a strict quantum benchmark, as the authors noticed.

Here we propose a benchmark based on a general phasecovariant family (pure or mixed): 


$$
\mathcal{F}=\int \frac{d \phi}{2 \pi} F\left(\rho_{\phi}, \rho_{\mathrm{av}}^{\phi}\right),
$$

where $F\left(\rho_{1}, \rho_{2}\right)=\left(\operatorname{tr} \sqrt{\sqrt{\rho_{1}} \rho_{2}} \sqrt{\rho_{1}}\right)^{2}=\left(\operatorname{tr}\left|\sqrt{\rho_{1}} \sqrt{\rho_{2}}\right|\right)^{2}$ is the fidelity, $\rho_{\phi}$ is the input test state defined as $\rho_{\phi}=U(\phi) \rho_{0} U(\phi)^{\dagger}$, with $U(\phi)=\sum_{n} e^{i \phi n}|n\rangle\langle n| \quad\left(|n\rangle\right.$ are Fock states), and $\rho_{\mathrm{av}}(\phi)$ $=\Sigma_{\chi} p\left(\chi \mid \rho_{\phi}\right) \rho_{\chi}$ is the state outputted by the measure-andprepare channel. In contrast to (ii) above [2,3], guessed states $\rho_{\chi}$ are not constrained to belong to the input-state family. In addition, since the fidelity is a convex function, a classical (measure and prepare) strategy that outputs a single state $\rho_{\mathrm{av}}$ provides always a higher average fidelity than any classical strategy that outputs different states $\rho_{\chi}$ depending on the outcome of the measurement. Therefore the fidelity in (i) [3] is arguably not a proper quantum benchmark. A benchmark based on phase-covariant states is appealing from an experimental point of view since phases are easy to vary without affecting other relevant parameters (in contrast, e.g., in the presence of losses, varying the degree of squeezing leads to a change in temperature of the test states).

Given a strategy characterized by positive operator-valued measure (POVM) elements $\left\{O_{\chi}=\left|\xi_{\chi}\right\rangle\left\langle\xi_{\chi}\right|\right\}$ (they can be taken to be of rank one without loss of generality and $\left|\xi_{\chi}\right\rangle$ are not normalized) and corresponding guesses $\left\{\rho_{\chi}\right\}$, one defines a covariant strategy by $\left\{O_{\chi, \theta}=1 /(2 \pi) U_{\theta} O_{\chi} U_{\theta}^{\dagger}\right\}$ and $\left\{\rho_{\chi, \theta}\right.$ $\left.=U_{\theta} \rho_{\chi} U_{\theta}^{\dagger}\right\}$. One can show that the optimal strategy can always be chosen to be covariant [10] and

$$
\mathcal{F}=\left(\operatorname{tr}\left|\sqrt{\rho_{0}} \sqrt{\rho_{\mathrm{av}}}\right|\right)^{2}, \quad \rho_{\mathrm{av}}=\int d \theta \sum_{\chi} p\left(\chi, \theta \mid \rho_{0}\right) \rho_{\chi, \theta} .
$$

Note that aside from the group parameter $\theta$, one needs to specify also the "seed" for both POVM $\left(O_{\chi}\right)$ and guess states $\left(\rho_{\chi}\right)$. For single-seed strategies, the completeness relation fixes the POVM, which turns out to correspond to the phase measurement [11]: $|\xi\rangle=\Sigma_{n}|n\rangle$ (up to some arbitrary phases).

The classical fidelity [Eq. (2)] can be conveniently written as

$$
\mathcal{F}=\max _{K}\left(\operatorname{tr}_{B} \sqrt{\operatorname{tr}_{A} \sqrt{\rho_{0}} \otimes \sqrt{\rho_{0}} K \sqrt{\rho_{0}} \otimes \sqrt{\rho_{0}}}\right)^{2},
$$

where $\operatorname{tr}_{A}$ ( similarly $\operatorname{tr}_{B}$ ) stands for the obvious partial trace and $K=\int d \theta \Sigma_{\chi} O_{\chi, \theta} \otimes \rho_{\chi, \theta}$. Optimizing the classical strategy amounts to maximizing the trace squared in Eq. (3) over the set of positive operators acting on $\mathcal{H} \otimes \mathcal{H}$ that are separable, invariant under bilateral transformations $U_{\theta} \otimes U_{\theta}$, and that fulfill $\operatorname{tr}_{B} K=\mathbb{1}_{A}$. For pure states, Eq. (3) can be simplified to give $\mathcal{F}=\left\langle\psi_{0}\left|\left\langle\psi_{0}|K| \psi_{0}\right\rangle\right| \psi_{0}\right\rangle$, with $\rho_{0}=\left|\psi_{0}\right\rangle\left\langle\psi_{0}\right|$. This leads to the pure-state estimation approach introduced in [12]. For a given POVM with seeds $\left\{\left|\xi_{\chi}\right\rangle\right\}$ the optimal fidelity can also be written as

$$
\mathcal{F}=\sum_{\chi} \sup _{\psi_{\chi}}\left\langle\psi_{\chi}\left|A_{\chi}\right| \psi_{\chi}\right\rangle=\sum_{\chi}\left\|A_{\chi}\right\|_{\infty},
$$

where $A_{\chi}=\int d \phi /(2 \pi)\left|\left\langle\xi_{\chi} \mid \psi_{\phi}\right\rangle\right|^{2}\left|\psi_{\phi}\right\rangle\left\langle\psi_{\phi}\right|$. Thus, if the POVM is fixed, the maximum fidelity is given by the largest eigenvalue of the operator $A_{\chi}$ and the optimal guess seed, $\left|\psi_{\chi}\right\rangle$, by the corresponding normalized eigenvector [1].
If one restricts the guess-states to be in $\Omega$ (as done in $[2,3])$, things simplify considerably, especially for pure states where no optimization is required since the optimal POVM is known to be the phase measurement [11]. In our case, no assumption about the optimal POVM nor about the guess is made, and we have to resort to more powerful techniques.

Semidefinite programming (SDP) [13] is an area of convex optimization that was developed in the last decade and that has recently found several applications in the field of quantum information $[12,14,15]$. Its aim is to minimize a linear objective function subject to semidefiniteness constraints involving symmetric matrices that are affine in the variables:

$$
\min _{x} \boldsymbol{c}^{T} \boldsymbol{x} \text { subject to } F(\boldsymbol{x})=F_{0}+\sum_{i} x_{i} F_{i} \geq 0,
$$

where $F_{i}$ are Hermitian matrices of arbitrary dimension and the inequality means that $F(\boldsymbol{x})$ is positive semidefinite. There are a number of freely available software packages to solve SDP problems. In this work we have used the YALMIP MATLAB toolbox [16] together with the SDPT3 solver [17].

For pure input states, the maximization in Eq. (3) can be immediately cast into a SDP problem by writing the matrices $K$ and $\rho_{0}$ in a basis of the Hermitian matrices. Whereas the positivity and bilateral invariance constrains are already in the desired form, the separability condition need be imposed through a hierarchy of constrains based on the positivity of the partial transpose (PPT) of all symmetric extensions [14]. In this work we will stick to the lowest level of this hierarchy imposing only PPT, i.e., $K^{\Gamma} \geq 0$. Since positive partial transposition provides a necessary but in general not sufficient condition for separability, the resulting optimal value, $\mathcal{F}^{\Gamma}$, gives an upper bound to $\mathcal{F}$ and hence still provides a valid quantum benchmark.

When the input test states are mixed, the objective function in Eq. (3) becomes truly nonlinear and the optimization problem does not immediately fall into the SDP category. However, one can make use of Uhlmann's theorem [18] and recast $\mathcal{F}$ in Eq. (2) as $\mathcal{F}=\max _{\Psi_{\text {av }}}\left|\left\langle\Psi_{0} \mid \Psi_{\text {av }}\right\rangle\right|^{2}$ $=-\min _{\sigma_{\mathrm{av}}}\left(-\left\langle\Psi_{0}\left|\sigma_{\mathrm{av}}\right| \Psi_{0}\right\rangle\right)$, where $\left|\Psi_{0}\right\rangle$ and $\sigma_{\mathrm{av}}=\left|\Psi_{\mathrm{av}}\right\rangle\left\langle\Psi_{\mathrm{av}}\right|$ are the purifications of $\rho_{0}$ and $\rho_{\mathrm{av}}$, respectively. Without loss of generality, the purity condition on $\sigma_{\mathrm{av}}$ can be lifted. With this, the objective function becomes a linear function of the optimization variables. Constraints are also of the SDP form in Eq. (5): (i) $\operatorname{tr}_{B} \sigma_{\mathrm{av}}=\rho_{\mathrm{av}}=\operatorname{tr}_{A}\left(\rho_{0} \otimes 1 K\right)$; (ii) $\sigma_{\mathrm{av}} \geq 0$ and $\operatorname{tr} \sigma_{\mathrm{av}}=1$; and (iii) the same conditions on $K$ as above.

\section{QUBIT STATES}

It will become apparent as we proceed that analytical solutions to the benchmark problem for general mixed states are exceedingly hard to obtain. A remarkable exception are qubit states, which we discuss next.

The input-state family is defined by $\rho_{\phi}=U_{z, \phi} \rho_{0} U_{z, \phi}^{\dagger}$, where $U_{z, \phi}$ is a rotation of an angle $\phi$ around the $z$ axis (similarly for $U_{y, \theta}$, etc.), and the seed input state is $\rho_{0}$ $=U_{y, \theta}\{p|\uparrow\rangle\langle\uparrow|+(1-p)| \downarrow\rangle\langle\downarrow|\} U_{y, \theta}^{\dagger}$ for fixed azimuthal angle $\theta$ and probability $p \equiv(1+\eta) / 2$ (so that $\eta$ is the modulus of the Bloch vector). To calculate the quantum benchmark for this 
family of qubit states, we use Eq. (3). In the up/down basis the most general $K$ can be written as $K=\operatorname{blockdiag}(a, B, c)$, where $B$ is a $2 \times 2$ positive semidefinite matrix and $a$ and $c$ are the non-negative numbers. For $C^{2} \otimes C^{2}$ the partial transposition criteria provides a necessary and sufficient condition for separability, i.e., $K$ is separable $\Leftrightarrow K^{\Gamma} \geq 0 \Leftrightarrow a c-\left|B_{12}\right|^{2}$ $\geq 0$. Finally, the condition $\operatorname{tr}_{B} K=1_{A}$ implies $a+B_{11}=c+B_{22}$ $=1$. A tedious but straightforward calculation leads to the optimal $K$ (that maximizes $\mathcal{F}$ ): $a=\cos ^{2}(\zeta / 2), c=\sin ^{2}(\zeta / 2)$, and $B_{12}=B_{21}=\sqrt{a c}$, where the (azimuthal) angle $\zeta$ is

$$
\zeta=\arctan \frac{\eta^{2} \sin ^{2} \theta+\sqrt{\left(1-\eta^{2}\right)\left(4-\eta^{2} \sin ^{2} \theta\right)}}{2 \eta \cos \theta} .
$$

It is a simple exercise to check that these values of $a, B$, and $c$ correspond to the phase-covariant POVM $\left\{1 / \pi U_{z, \phi} U_{y, \frac{\pi}{2}}|\uparrow\rangle\langle\uparrow| U_{y, \frac{\pi}{2}}^{\dagger} U_{z, \phi}^{\dagger}\right\}$, with the associated guess $|\phi\rangle=U_{z, \phi} U_{y, \zeta}|\uparrow\rangle$. The resulting benchmark for qubit states can be cast as

$$
\mathcal{F}=\frac{1}{2}\left(1+\eta \frac{\cos \theta}{\cos \zeta}\right) .
$$

Some remarks are in order: (i) for any outcome $\phi$ the corresponding guess is a pure state and, hence, (ii) it does not belong to the original family; moreover, (iii) its Bloch vector is not proportional to that of the signal state $(\zeta \neq \theta$, unless $\theta=\pi / 2$ ). In quantum state estimation it is usually assumed that the guess after a measurement is one of the possible input states. Our result shows that this assumption is not always well founded. (iv) Recalling the definition of $K$ right after Eq. (3), we see that only one value of $\chi$ (a single seed) is required for qubits. Property (iv) is specific of $\mathbb{C}^{2}$. For $\mathbb{C}^{4}$ one can already find examples where the phase measurement is not optimal.

We now move to the benchmarks for CV Gaussian states. We consider displaced and squeezed thermal states that are obtained by the action of the displacement, $D(\alpha)$ $=\exp \left[\alpha\left(a^{\dagger}-a\right)\right]$, and the squeezing, $S(r)=\exp \left[\frac{r}{2}\left(a^{2}-a^{\dagger 2}\right)\right]$, operators over a thermal state $\rho_{\beta}=\left(1-e^{-\beta}\right) e^{-\beta a^{\dagger} a}$ of purity $\mu=\tanh \beta / 2$ (with $\alpha>0$ and $r>0$ ).

\section{CV PURE STATES}

We start by computing the bound $\mathcal{F}^{\Gamma}$ using the SDP approach for coherent states. For this purpose we use a truncated Fock basis and approximate low amplitude coherent states by $|\alpha\rangle \approx e^{-\alpha^{2} / 2} \sum_{n=0}^{N} \alpha^{n} / \sqrt{n !}|n\rangle$. Figure 1 (dots) shows the results for coherent states with mean photon number between zero and $\alpha^{2}=10$ (the truncation error within this range of $\alpha$ and $N=23$ is always lower than $10^{-4}$ ). In addition we can calculate the optimal fidelity when restricting to the single-seed covariant POVM. With this choice of POVM the problem reduces to calculating the maximum eigenvalue of

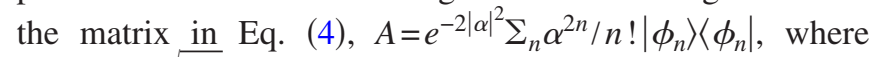
$\left|\phi_{n}\right\rangle=\sum_{l=0}^{n} \sqrt{\left(\begin{array}{c}n \\ l\end{array}\right)}|l\rangle$. The values for different input intensities are shown in Fig. 1 (solid line) and agree with those obtained from the SDP optimization. This indicates that the benchmark given by $\mathcal{F}^{\Gamma}$ is attainable with the phase measurement
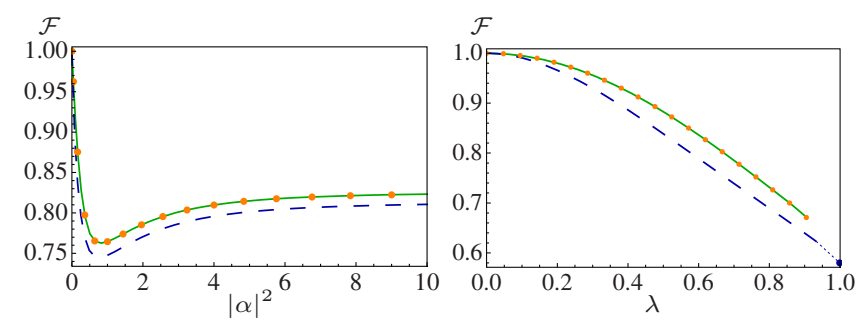

FIG. 1. (Color online) Plot of the fidelity for pure coherent states (left) and pure squeezed states (right). Dots: bound obtained imposing $K^{\Gamma} \geq 0$ (see main text). Solid: phase measurement and optimal guess. Dashed: phase measurement and guess from input family. Dotted: extrapolation of dashed line to infinite squeezing, $\lambda=1$.

(at least within the precision of our numerical analysis). We note that the eigenvector of $A$ with largest eigenvalue, which is the optimal guess, resembles a coherent state but is strictly different. The dashed line in Fig. 1 is the fidelity obtained when the guess is forced to be a coherent state, and although it has a similar behavior, it shows a clear gap with the optimal bound. In this case no optimization is required and $\mathcal{F}$ can be obtained by numerical integration of

$$
\mathcal{F}=\int \frac{d \phi}{2 \pi}\left|\left\langle\xi \mid \alpha e^{i \phi}\right\rangle\right|^{2}\left|\left\langle\alpha \mid \alpha e^{i \phi}\right\rangle\right|^{2} .
$$

From the above equation it is possible to find the analytical value of $\mathcal{F}$ in the limit $\alpha \rightarrow \infty$. In this regime, the outcome probabilities can be approximated by $\left|\left\langle\xi \mid \alpha e^{i \phi}\right\rangle\right|^{2}$ $\simeq \sqrt{2 \alpha^{2} / \pi} \exp \left[-2 \alpha^{2} \phi^{2}\right]$, where we have used the Gaussian limiting expression of a Poisson distribution, $\left|\left\langle\alpha \mid \alpha e^{i \phi}\right\rangle\right|^{2}$ $\approx \exp \left[-\alpha^{2} \phi^{2}\right]$, and extended the range of integration to $(-\infty, \infty)$ to find $\mathcal{F}_{\alpha \rightarrow \infty}=\sqrt{2 / 3}$.

The analytical value of $\mathcal{F}$ in the large $\alpha$ limit can also be obtained in the general case of unrestricted guess states. In this case one has to calculate the maximum eigenvalue of $A$ defined in Eq. (4), which can be done by calculating the limit $p \rightarrow \infty$ of its $p$ norm $\|A\|_{p}=\left(\operatorname{tr} A^{p}\right)^{1 / p}$. We have

$$
\left(\|A\|_{p}\right)^{p}=\operatorname{tr} A^{p}=\int \prod_{j=1}^{p} d \phi_{j} p\left(\chi \mid \phi_{j}\right)\left\langle\alpha_{j} \mid \alpha_{j+1}\right\rangle,
$$

where $\alpha_{p+1} \equiv \alpha_{1}$. Using the above approximation on the outcome probabilities and $\left\langle\alpha_{i} \mid \alpha_{j}\right\rangle$ $\approx \exp \left\{-\alpha^{2}\left[i\left(\phi_{i}-\phi_{j}\right)+1 / 2\left(\phi_{i}-\phi_{j}\right)^{2}\right]\right\}$, we obtain

$$
\operatorname{tr} A^{p} \simeq\left(\frac{2 \alpha^{2}}{\pi}\right)^{p / 2} \int d^{p} \phi e^{-\alpha^{2} / 2 \phi^{t} C_{p} \phi}=\frac{2^{p}}{\sqrt{\operatorname{det} C_{p}}},
$$

where $C_{p}$ is a symmetric matrix with elements $\left[C_{p}\right]_{i j}$ $=6 \delta_{i j}-\delta_{i+1, j}-\delta_{i, 1} \delta_{j, p}$ for $i \leq j$. It is convenient to write $C_{p}$ $=M_{p}+2 a \rrbracket_{p}$, with $a=3$. Then, $\operatorname{det}\left(M_{p}+2 a \rrbracket_{p}\right)=Q_{p}(a)$ is a (characteristic) polynomial in $a$. It easy to check that $Q_{p}(a)$ $=2\left[T_{p}(a)-1\right]$, where $T_{p}(a)=\left[\left(a+\sqrt{a^{2}-1}\right)^{p}+\left(a-\sqrt{a^{2}-1}\right)^{p}\right] / 2$ are the Chebyshev polynomials of the first kind. In the limit $p \rightarrow \infty$ the second term in $T_{p}(a)$ becomes negligible, hence $Q_{p}(3) \approx(3+\sqrt{8})^{p}=(\sqrt{2}-1)^{2 p}$, and 


$$
\mathcal{F}=\lim _{p \rightarrow \infty} 2\left(\operatorname{det} C_{p}\right)^{1 / 2 p}=2(\sqrt{2}-1) \approx 0.8284,
$$

which indicates that the difference between the fidelities corresponding to restricted (guess in $\Omega$ ) and unrestricted (general guess) strategies persists also in the asymptotic regime.

For pure squeezed states we proceed as before by working in a truncated basis: $|\lambda\rangle$ $=\left(1-\lambda^{2}\right)^{1 / 4} \sum_{n=0}^{N}(-\lambda / 2)^{n} \sqrt{(2 n) !} / n !|2 n\rangle$. Figure 1 shows the SDP results, together with the bound obtained from the phase measurement with optimal guess (the maximum eigenvalue of $A$ ) and from the phase measurement with (restricted) squeezed guess. The latter is obtained by numerically integrating

$$
\mathcal{F}=\int \frac{d \phi\left(1-\lambda^{2}\right)^{2}}{\sqrt{1+\frac{4 \lambda^{2}}{\left(1-\lambda^{2}\right)^{2}} \sin ^{2} \phi}}\left|\sum_{n} \frac{\sqrt{(2 n) !}}{n !}\left(\frac{\lambda e^{2 i \phi}}{2}\right)^{n}\right|^{2} .
$$

The SDP bound and the phase-measurement fidelity agree within numerical precision. As for coherent states, restricting the guess states be in $\Omega$ lowers the bound substantially. The latter bound can again be computed in the limit $\lambda \rightarrow 1$ by noticing that the dominant behavior of the sum in Eq. (12) is dictated by the large $n$ terms [19]. Using the Stirling's approximation, the sum in Eq. (12) can be written as a polylogarithm function $\operatorname{Li}_{1 / 4}\left(\lambda e^{2 i \phi}\right) / \pi^{1 / 4}$. Taking into account that the dominant contribution to the integral comes from the region $\phi \sim 0$, we get $\mathcal{F} \simeq 0.58$.

\section{CV MIXED STATES}

The case of mixed states is remarkably more complex, and we have to entirely rely on numerical analysis. Figure 2 shows the SDP results obtained for displaced thermal states
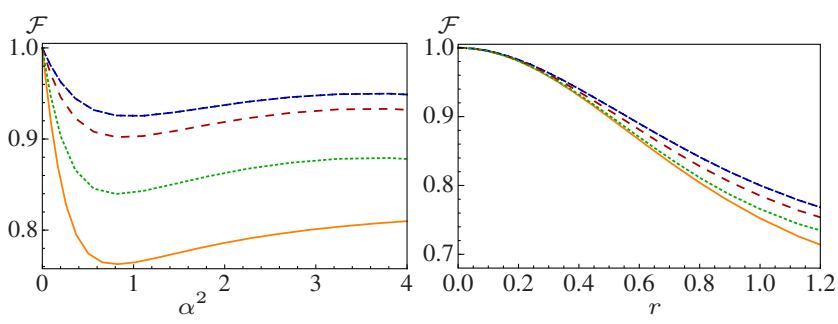

FIG. 2. (Color online) Benchmarks for displaced (left) and squeezed (right) thermal states for different purities: $\mu=1$ (solid line), $\mu=0.95$ (dots), $\mu=0.8$ (dash), and $\mu=0.7$ (dash-dot).

and squeezed thermal states of different purity $\mu$ [20]. The truncation errors at the higher values of $r$ and $\alpha^{2}$ are of the order of a few percent, but the displayed values still provide a good upper bound because truncation effects tend to lower the curves. We observe that decreasing the purity has the effect of increasing the fidelity. Thus our benchmark is especially suited for test states of moderate temperature. It is worth mentioning here that if the guess is restricted to belong to the input family (not shown in plot), the effect is the opposite: pure states provide higher fidelities than mixed states.

Note added in proof: Recently, analytical results after the completion of this work, as well as the SDP reduction, have been shown [21] for a similar quantum benchmark, which is however not based on fidelity.

\section{ACKNOWLEDGMENTS}

We are grateful to G. Adesso and G. Chiribella for discussions. We acknowledge financial support from the Spanish MICINN through the Ramón y Cajal program (J.C.), Grants No. FIS2005-01369 and No. FIS2008-01236/FIS, and QOIT (Consolider-Ingenio 2010), and from the Generalitat de Catalunya under Grant No. CIRIT SGR-00185.
[1] K. Hammerer et al., Phys. Rev. Lett. 94, 150503 (2005).

[2] A. Serafini et al., Phys. Rev. Lett. 98, 170501 (2007).

[3] G. Adesso and G. Chiribella, Phys. Rev. Lett. 100, 170503 (2008).

[4] A. Furusawa et al., Science 282, 706 (1998).

[5] B. Julsgaard et al., Nature (London) 432, 482 (2004); K. Honda et al., Phys. Rev. Lett. 100, 093601 (2008); J. Appel et al., ibid. 100, 093602 (2008).

[6] T. C. Ralph and P. K. Lam, Phys. Rev. Lett. 81, 5668 (1998).

[7] H. Häseler et al., Phys. Rev. A 77, 032303 (2008).

[8] S. L. Braunstein et al., J. Mod. Opt. 47, 267 (2000).

[9] D. Bruß and C. Macchiavello, Phys. Lett. A 253, 249 (1999).

[10] Starting from definition (1) and using the properties: (i) for any two unitaries $U$ and $V$ the trace norm satisfies $\operatorname{tr}|U B V|=\operatorname{tr}|B|$; (ii) the fidelity is convex, and (iii) $U(\phi) U(\theta)^{\dagger}=U(\phi-\theta)$.

[11] A. S. Holevo, Probabilistic and Statistical Aspects of Quantum Theory (North-Holland, Amsterdam, 1982).

[12] M. Navascues, Phys. Rev. Lett. 100, 070503 (2008).

[13] L. Vandenberghe and S. Boyd, SIAM Rev. 38, 49 (1996).
[14] A. C. Doherty et al., Phys. Rev. A 69, 022308 (2004).

[15] E. Rains, IEEE Trans. Inf. Theory 47, 2921 (2001); K. Audenaert et al., Phys. Rev. Lett. 87, 217902 (2001); K. Audenaert and B. De Moor, Phys. Rev. A 65, 030302(R) (2002); M. Ježek et al., ibid. 65, 060301(R) (2002); J. Eisert et al., ibid. 70, 062317 (2004); A. S. Fletcher et al., ibid. 75, 012338 (2007); M. Navascués et al., New J. Phys. 10, 073013 (2008).

[16] J. Löfberg, Proceedings of the CACSD Conference, Taipei, Taiwan, 2004 (unpublished).

[17] K. C. Toh et al., Optim. Methods Software 11, 545 (1999).

[18] A. Uhlmann, Ann. Phys. 497, 524 (1985).

[19] E. Bagan et al., Phys. Rev. A 78, 043829 (2008).

[20] The results for single-seed POVM, which are not shown in the figure, do not coincide with the general bound for squeezed thermal states but follow practically the same curve. For displaced thermal states, the single-seed POVM does attain the general bound.

[21] M. Owari et al., New J. Phys. 10, 113014 (2008). 